\title{
Modeling Self-Healing Behavior of Vitrimers using Molecular Dynamics with Dynamic Cross-Linking Capability
}

\author{
Gurmeet Singh $^{\mathrm{a}}$, Veera Sundararaghavan ${ }^{\mathrm{a}, *}$ \\ ${ }^{a}$ Department of Aerospace Engineering, University of Michigan, Ann Arbor MI 48109, \\ U.S.A.
}

\begin{abstract}
Vitrimers are a special class of polymers that undergo dynamic cross-linking under thermal stimuli. Their ability to exchange covalent bonds can be harnessed to mitigate damage in a composite or to achieve recyclable aerospace composites. This work addresses the primary challenge of modeling dynamic cross-linking reactions in vitrimers during thermomechanical loading. Dynamic bond exchange reaction probability change during heating and its effect on dilatometric and mechanical response are simulated for the first time in large scale molecular dynamics simulations. Healing of damage under thermal cycling is computed with mechanical properties predicted before and after self-healing.
\end{abstract}

Keywords: Vitrimer, dynamic cross-linking, nanocomposite, self-healing, CNT, covalent adaptable networks (CANs)

Please find and cite the published version of the article here:

Gurmeet Singh and Veera Sundararaghavan "Modeling self-healing behavior of vitrimers using molecular dynamics with dynamic cross-linking capability", Chemical Physics Letters 760 (2020) 137966.

DOI $=$ doi.org/10.1016/j.cplett.2020.137966

\footnotetext{
${ }^{*}$ Corresponding author

Email address: veeras@umich.edu, Tel: +1 (734) 615-7242 (Veera Sundararaghavan)
}

Preprint submitted to Chemical Physics Letters

December 25, 2020 


\section{Introduction}

Thermoset polymers find applications in fields ranging from robotics, aerospace, automobile, electronics, and batteries either as is or as a part of fibrous composites [1. There are some challenges in their usage that need to be addressed, for instance, the inability to recycle and re-process due to the irreversibility of the cross-linking bonds and damage evolution in structurally loaded components. Vitrimers are a promising alternative material system that has been recently designed to address these issues $2,3,3,4,5,6$. 6. Vitrimers contain dynamic cross-links that enable them to behave like thermosets at low temperatures and behave like thermoplastics at higher temperatures [7. This enables self-healable aerospace composites where damage can be reversed through heating, or recyclable matrix materials where the matrix can be reclaimed after use [8, 9, 1]. Numerous covalent bond exchange mechanisms have been discovered in the recent past such as amines [10, 11, transesterification reactions 12], Diels-Alder reaction [13, radical formation [6], etc. Vitrimers have been recently incorporated in carbon fiber composites [14, 3] and nanocomposites [15] which expands their scope of applications.

Molecular dynamics (MD) models of conventional thermosets have now found use in the aircraft industry. In recent years, these models have demonstrated reliable prediction of the glass transition temperature [16, gelation point [17, thermal expansion coefficient [18, thermal conductivity [19, 20], elastic properties 21, and even complete yield surface 22 without any experimental inputs allowing for computational materials design. The primary challenge for vitrimers is the presence of temperature dependent reversible cross-link reactions that dynamically alter the mechanical response. Exchange reactions have been modelled in the past via methods such as embedding Monte Carlo (MC) moves into molecular dynamics (MD) or fully MD (using specialized three body potentials) or fully MC simulations to simulate bond swaps [23, 24, 25, 26, 27. These simulations have typically employed coarse grained models (bead-spring) that provide high computational efficiency while approximating the mechanical response. For more quantitative modelling, all-atom MD methods are attractive 28, 29, however these methods become computationally demanding when simulating slow chemical and mechanical dynamics. Yang et al. 30] modelled bond exchange reactions in all-atom MD by implementing a distancebased reaction cutoff, which greatly accelerates the chemical dynamics. Bonds were created based on the proximity of reacting atoms and the topology was accepted based on the energy of the new bond. However, the simulation had to be started with a low distance cutoff for stability considerations (to avoid large changes in energies due to initial reactions) and the cutoff was subsequently increased. In this paper, we avoid this issue using an algorithm for chemical reactions based on a pre and post-reaction templates with fixed proximity cutoff 31, 32. Our approach employs an explicit temperature dependence of reaction probabilities drawn from experimental insights. The approach allows, for the first time, modeling of mechanical property changes in vitrimers during thermal cycling above and below topology freezing point $(T v)$ while demonstrating 
(a)

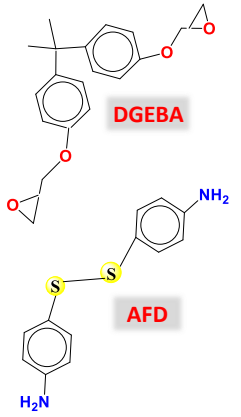

(b)

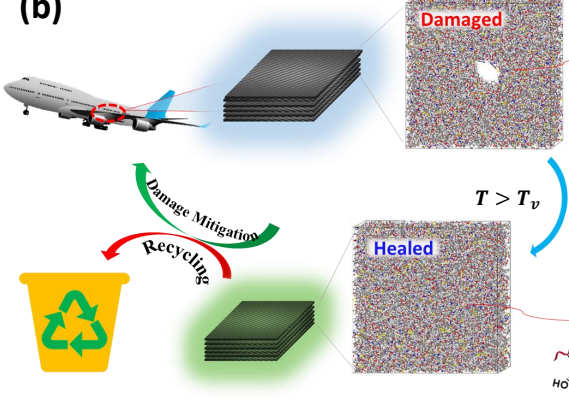

(c)

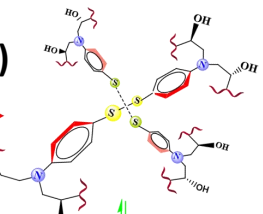

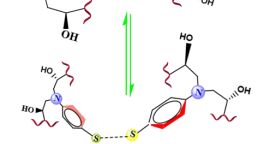

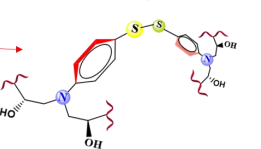

Figure 1: (a) Vitrimer monomer units, (b) shows the importance of vitrimer based fibrous composites towards damage mitigation and recycling during operational cycles via (c) the dynamic di-sulfide bond exchange reaction

healing of damage and subsequent recovery of mechanical properties.

In this work, we considered epoxy monomer diglycidyl ether of bisphenol A (DGEBA) cross-linked with 4-aminophenyl disulfide (AFD) vitrimer (structures shown in Fig. 1(a)). This particular system was chosen due to its ability to achieve dynamic cross-links in the absence of catalysts 33. This isomer of AFD also demonstrates mechanochromatic behavior within the visible spectrum (due to the position of amine group at ortho position 34] which is useful for damage detection. Further, this is one of the few vitrimeric systems that has been integrated into a fiber composite [35. Through reconfigurable sulfur-sulfur (S-S) linkage (Fig. 1(c)), the network can change its topology, preserving the number of bonds but at the same time relaxing its stresses. When the temperature decreases (cooling), the exchange reactions slow down and the network topology appears to be fixed on experimental time scales. Hence, it behaves like an elastic thermoset (elastomer). Thermoset composites are prone to damage during operation and which diminishes their performance (shown in Fig. 11(b)). Upon heating vitrimers above the topology freezing point, the dynamic bond exchange reactions accelerate (Fig. 11(c)) and the viscosity decreases due to preference towards linear chains, causing the vitrimer to become malleable. Such behavior can be used to heal damage and to recover elastic properties upon cooling back to temperatures below the topology freezing point 34. In this paper, we have employed a temperature dependent probability model for the dynamic di-sulfide bond exchange reaction to simulate this effect.

\section{Methods}

We started with a simulated cell containing a monomer mixture with DGEBA:AFD. The typical synthetic epoxy to hardener stoichiometric ratio of 2:1 was employed [36. We repeat this unit by $8 \times 8 \times 8$ to get a simulation box with 2,048 DGEBA and 1,024 AFD units, with a total of 68,608 atoms. Consistent valence force 
field (cvff) is assigned to all the atoms with pair, bond, angle, dihedral and improper coefficients modeled 37]. The non-bonded interactions are modeled using Lennard-Jones (LJ) and Coulombic pairwise interaction with a cutoff. The mixture is compressed to a liquid density of $1.0 \mathrm{gcm}^{-3}$ at $300 \mathrm{~K}$ and $1 \mathrm{~atm}$ NPT. Then the mixture is cured via curing reaction modeling in LAMMPS 32 . This is not a force-field based reaction modeling, but a bonding procedure for two atoms which mimics a chemical reaction 31 and the sites are identified by the pre- and post- reaction templates as well as on the mapping between the two templates 31] (a schematic shown in Figure S1 of supplemental information (SI)). The primary and secondary amine reactions are modeled with their respective reaction templates as well as their reaction maps (refer Figure S2 of $\mathrm{SI})$. The cut off distance between $\mathrm{C}$ and $\mathrm{N}$ atoms is set to be $3.5 \AA$ and 5.0 $\AA$ for the primary and secondary amine reactions, respectively. In addition to bonding cutoff distance, a reaction probability of one was assigned for the curing reaction. The system is able to achieve up to $95 \%$ cross-linking density (Figure S3, S4 of SI). The cured model is then annealed by heating and cooling cycles at $1 \mathrm{~K}$ (below $T_{g}$ ) and $600 \mathrm{~K}$ (well above $T_{g}$ ) under NPT conditions at 1 bar until the density converges to a $\rho=1.18 \mathrm{~g} / c c$ for neat vitrimer at $1 \mathrm{~K}$ (refer Figure S5 of SI). The equilibrated structure is used to further study thermo-mechanical properties and self-healing behavior under dynamic S-S bond exchange processes.

The dynamic S-S bond exchange process is modeled in terms of two step reactions. A pre and post-reaction templates are constructed for both reactions along with a reaction map (refer Figure S6 of SI for reaction templates). When two pairs of di-sulfide sites come together, they can exchange the chains attached to them as shown in Fig. 1.(c). The bond exchange reaction can happen when the distance between any sulfur atoms from different chains come within a cutoff distance of $4.12 \AA$ (double of the S-S bond length $38,2.06 \AA$ ) and when such sites are identified, the probability of the reaction is modeled as a function of temperature. For vitrimers, the temperature at which the acceleration of the reaction rate occurs is referred as topology freezing temperature $\left(T_{v}\right)$ and this value can be different or close to the glass transition temperature $\left(T_{g}\right)$ of the vitrimer [4, 39]. In this paper, we assumed both the transitions occur at the same temperature $\left(T_{v}=T_{g}=403 \mathrm{~K}\right)$. However, in case of different transition temperatures, the vitrimer behavior can be modeled by considering $T_{v}$ as the reference for dynamic bond exchange reaction probability. It is observed in experiments that the dynamic bond exchange reactions are very slow at room temperature but can accelerate near or above the topology transition $\left(T_{v}\right)$ temperature of this vitrimer system [33, 34]. The modulus is seen to soften and the mobility of the chains accelerate around the transition temperature in a sigmoidal manner 40. Based on this insight, we model the topology transition by accelerating the dynamic bond exchange reactions in a sigmoidal manner around the vitrimer transition temperature. Note that the actual experimental time scales of the exchange reactions are slower 41, and accelerated rates serve to realistically capture the thermomechanical behavior within the time scale 
of molecular dynamics simulations 28, 29]. To model the onset of dynamic exchange reaction phenomenon in the current model, we assign the probability of $\mathrm{S}-\mathrm{S}$ bond exchange reaction as a function of the temperature as shown in Fig. 2.

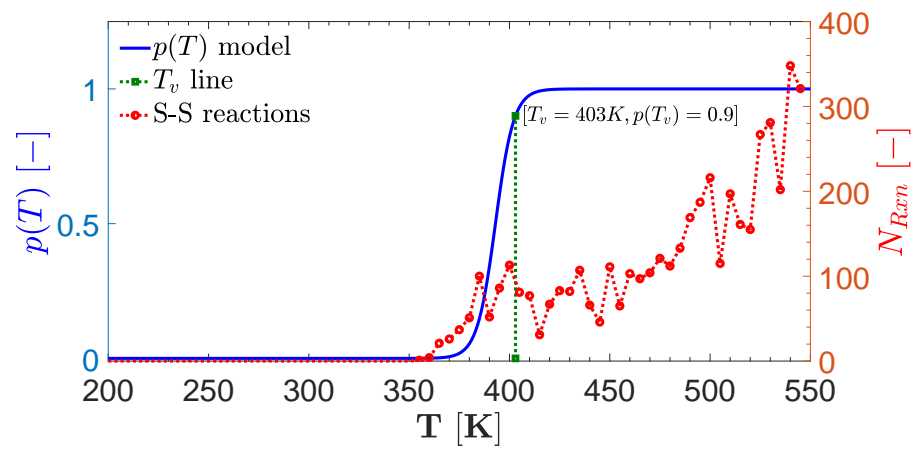

Figure 2: The reactions probability and the resulting number of $\mathrm{S}-\mathrm{S}$ bond exchange reactions $\left(N_{R x n}\right)$ vs. temperature

The probability of the dynamic bond reaction as a function of temperature is given by Equation 1 .

$$
p(T)=\frac{1}{\left[\exp \left(-a\left(T-T_{v}\right)-\ln \left(\frac{p_{T_{v}}}{1-p_{T_{v}}}\right)\right)+1\right]}
$$

where, $a=\frac{2 p_{T_{v}}}{w\left(1-p_{T_{v}}\right)}$ is determined by $w$ which is the measure of the width of the transition from glassy to rubbery phase measured, for example, using the modulus vs temperature response (we consider, $w=20 \mathrm{~K}$ ), and $p_{T_{v}}=0.9$ is the probability of the dynamic bond exchange reaction at $T_{v}\left(T_{v}\right.$ line in Fig. 2), we assume that at $T_{v}$, most of the transition starts occurring but it is not complete hence $p_{T_{v}}=0.9$. And, we assume that at the beginning of the transition window, $T_{v}-w$, the probability is $p\left(T_{v}-w\right)=1.0-p_{T_{v}}=0.1$. This is considered in order to obtain a smooth increase in the reaction rate near $T_{v}$.

\section{Results and Discussion}

We first analyze the molecular dynamics model without dynamic S-S bond (indicated in plots as 'static' model) and with dynamic S-S bond exchange modeled. The annealed structure is equilibrated for 150ps 42] at each temperature starting from $200 \mathrm{~K}$ to $550 \mathrm{~K}$ at an increment of $5 \mathrm{~K}$. The change in volume is computed and normalized with respect to the initial volume at $200 \mathrm{~K}\left(V_{0}\right)$. The algorithm keeps a cumulative count of bond exchange reactions that occurred in the system 31. In this system, the number of bonds remain conserved, and hence, bond breaking is accompanied with new S-S bond formation (as sketched in Fig 1(c)). The number of S-S bond-exchange reactions occurring at each temperature is depicted as a red line in Fig. 2 alongside the chosen reaction 
probability (in blue). This plot indicates that the number of reactions begin to increase as we get close to $T_{v}$ and stabilizes around $T_{v}$. At temperatures well beyond $T_{v}$ (when the probability of reaction is 1.0), the number of reactions increase further with increase in temperature. This is attributed to an increase in the frequency of collision events of bonding atoms at higher temperatures. These features are achieved by developing a reaction probability centered around the topology freezing transition point based upon experimental insights 33,34 . If the vitrimer transition temperature were lower than the glass transition temperature, then the reaction rates will be lower owing to higher stiffness of the polymer which will significantly decrease the collision frequency. As an example, we find that the number of exchange reactions over 150 ps was $53 \%$ lower for $T_{v}$ set at $200 \mathrm{~K}$ vs $403 \mathrm{~K}$ for this polymer. By changing the $T_{v}$ value, we expect the model to capture the topology transition behavior for other such vitrimers as well.

Fig. 3 shows the V-T characteristic of the neat vitrimer for static and dynamic S-S bond modeled in the simulation. The plot clearly shows that there is no change in the volume expansion for dynamic and static S-S bond until the reactions start picking up near $T_{v}-w=403 \mathrm{~K}-20 \mathrm{~K}=383 \mathrm{~K}$ when the probability of the reaction is $1-p_{T_{v}}=0.1$. We also observe that the dynamic $\mathrm{S}-\mathrm{S}$ bond model has a higher coefficient of thermal expansion as compared to the static S-S model in the rubbery region beyond $T_{v}$. This is expected due to the added mechanical flexibility owing to dynamic rearrangements of chains 2. The static coefficient of thermal expansion below $T_{v}$ of $41.1 \pm 2.7 \mu K^{-1}\left(=\frac{\Delta V}{3 V_{o}}\right)$ falls at the lower end of range reported $\left(45.0-65 \mu \mathrm{K}^{-1}\right)$ for cured epoxy resins [43]. The model is next employed in a first attempt in literature, to the best of authors knowledge, to understand the effect of dynamic bond exchange reaction on mechanical behavior at different temperatures.

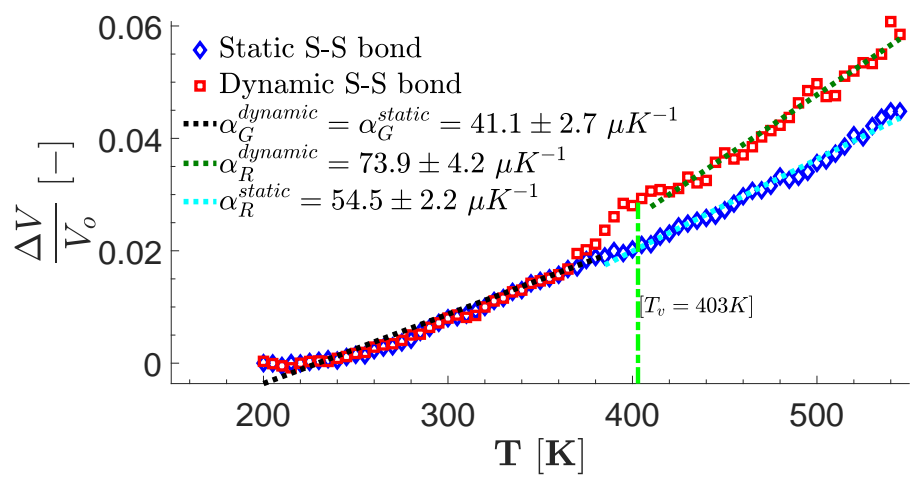

Figure 3: Volumetric expansion vs. T for model with and without modeling S-S bond reactions. $\mathrm{R}$ and $\mathrm{G}$ represent rubbery and glassy phases of the glass transition, respectively.

In Fig. 4. stress-strain relationship as a result of stretching the simulation box along an axis at a strain rate of $2 \times 10^{8} s^{-1}$ at different temperatures is shown. At higher temperatures, both models show softening behavior. However, the 


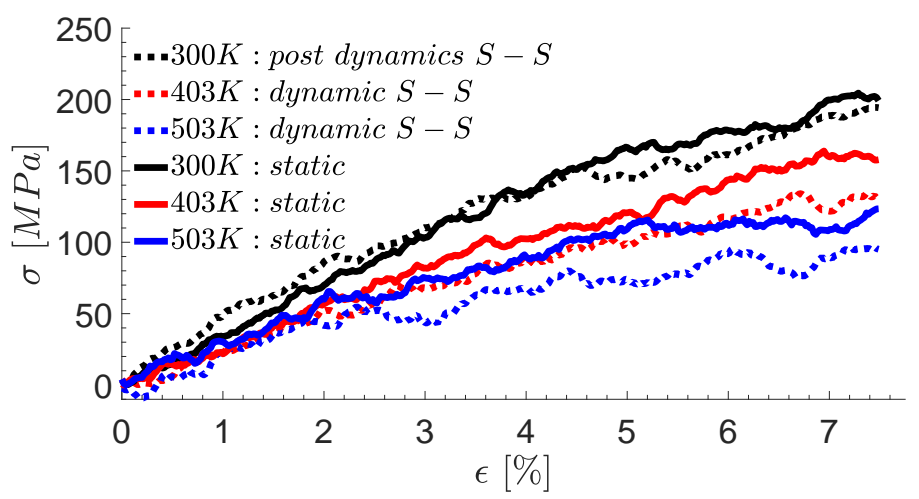

Figure 4: Influence of dynamic S-S bond modeling under uni-axial tension

difference in the stress-strain behavior is pronounced at high temperatures $\left(T_{v}+\right.$ $100 \mathrm{~K}$ ) where dynamic bond exchange dominates and vitrimeric material remains softer. The dynamic bond exchange model is taken to $503 \mathrm{~K}$ for $250 \mathrm{ps}$ and relaxed back to $300 \mathrm{~K}$ to compare the room temperature behavior after thermal cycling. In this case, the post dynamic bond and the static $\mathrm{S}-\mathrm{S}$ bond cases follow each other well, which shows that despite the model having undergone dynamic bond exchange reactions upon heating, the elastic response is retained at room temperature $(300 \mathrm{~K})$. This is inline with the understanding of the response of the recycled vitrimers [4, 5]. Note that due to inherently high strain rates employed in molecular dynamics, the Young's modulus of DGEBA:AFD vitrimer remains overpredicted $(4.5 \pm 0.21 \mathrm{GPa})$ compared to experiment (2.6 GPa) under quasistatic loading 35] conditions at 300K. MD simulations have predicted in the range of $3.4 \mathrm{GPa}$ to $5.8 \mathrm{GPa}$ for epoxies 44, 45, 46, 47 in literature. Note that while a vitrimer behaves like a viscous fluid beyond the topology transition temperature $\left(T_{v}\right)[3,14,15$, molecular dynamics results show sustained stresses beyond $T_{v}$ due to the high loading rates (as is also seen in MD literature [28, 29]).

We now exploit the dynamic S-S bond exchange reaction capability to demonstrate healing of vitrimers in MD simulations. The damage corresponds to a carbon nanotube (CNT) pullout from the matrix 48, 49, 50. We first insert a $(12,12)$ single walled CNT of $91 \AA$ length along $z$-axis, and displace the atoms radially in $x y$ plane. The rest of the curing protocol, described in the methods section, remains same as the neat vitrimer model with a final density of $\rho=1.19 \mathrm{gcm}^{-3}$ at $1 \mathrm{~K}$ (refer Figure S5 of SI). To generate the damage, the CNT was removed out of the equilibrated simulation box. Then the simulation box is heated up to a temperature $T_{v}+100 \mathrm{~K}=503 \mathrm{~K}$ for $250 \mathrm{ps}$. The hole heals under the influence of dynamic S-S bond exchange reactions. The healed system is then relaxed back to analyze its elastic response at room temperature. Fig. 5 shows the elastic response of the damaged and healed vitrimers (averaged of three different direction stretches with the bounds shown by light colors) at $300 \mathrm{~K}$. We observe that the stress-strain response of the healed vitrimer is consistently higher than that of the initial damaged sample along all three loading 
directions demonstrating healing. In order to plot the elastic properties over a range of temperatures, we computed the stiffness by linear regression in $\epsilon=4 \%$ range and the elastic modulus was averaged in all three directions. Fig. 6 demonstrates that the healed structure is able to recover the pristine vitrimer elastic modulus over the range of the temperatures below topology freezing point. Snapshots of damage healing showing the hole left by CNT pullout being filled via a dynamic bond exchange mechanism post $T_{v}$ are shown as inset (refer animation in SI).

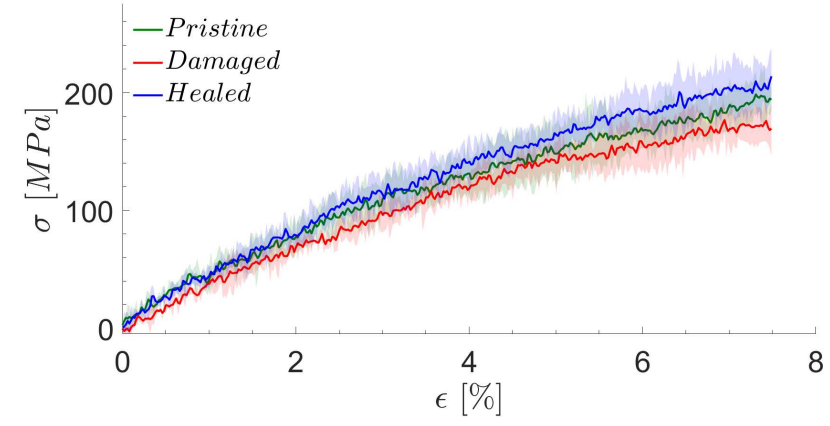

Figure 5: Stress-strain response under uniaxial tension in different directions at $300 \mathrm{~K}$ for damaged and healed samples.

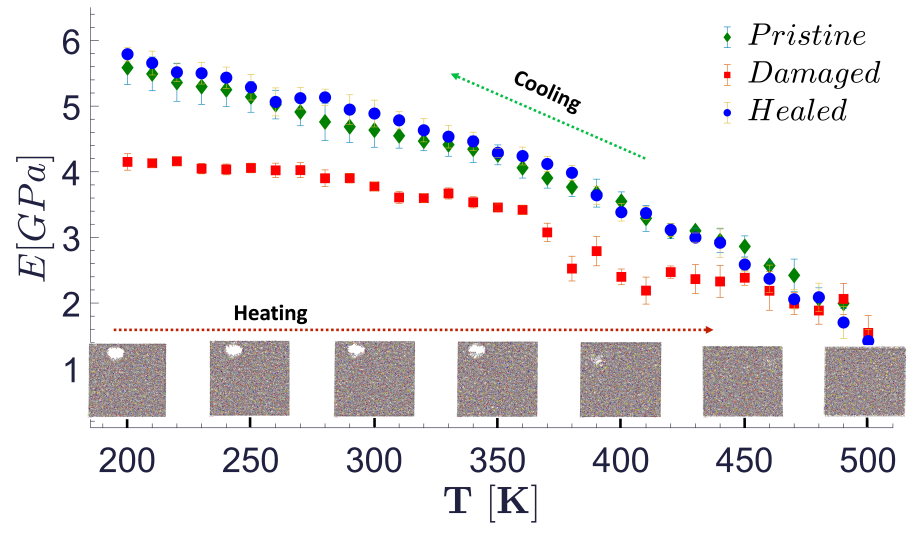

Figure 6: Stiffness enhancement post damage healing via S-S dynamic bond exchange reactions. 


\section{Conclusions}

In conclusion, a vitrimer's ability to dynamically reform covalent bonds during thermal cycling allows one to achieve superior behavior over traditional thermosets such as damage healing. To model this behavior, we have developed a novel temperature dependent reaction probability which is integrated with molecular dynamics modeling of vitrimers. The reaction probability is empirically based on the observed reaction kinetics of dynamic bond exchange near or above topology freezing transition temperature $\left(T_{v}\right)$. It is shown that the model captures the onset as well as the increase in number of reactions above this transition point owing to higher mobility of chains, without the need to alter reaction distance cutoffs. The vitrimer is seen to achieve softer behavior around and beyond topology freezing transition temperatures while maintaining the glassy behavior at pre-transition temperatures upon thermal cycling. In the simulation of the healing of a cylindrical pore created due to CNT pullout, the model is able to show both the healing of the vitrimer and complete recovery of elastic modulus upon cooling. Such modeling capability can be further used to achieve insights into the interplay of mechanics and chemistry in a variety of other dynamic bond exchange materials.

\section{Acknowledgement}

This research was supported in part through computational resources and services provided by Advanced Research Computing at the University of Michigan, Ann Arbor. The authors would like to thank Mr. Siddhartha Srivastava for his valuable discussions.

\section{Supplementary Information}

Supplementary material contains additional data on curing of the polymer mixture, the annealing protocol, and pre and post-reaction templates of the dynamic bond exchange reaction and an animation of damage healing via dynamic bond exchange.

Data Availability Statement: The data that support the findings of this study are available from the corresponding author upon reasonable request.

\section{References}

[1] M. K. Mcbride, B. T. Worrell, T. Brown, L. M. Cox, N. Sowan, C. Wang, M. Podgorski, A. M. Martinez, C. N. Bowman, Enabling Applications of Covalent Adaptable Networks, Annu. Rev. Chem. Biomol. Eng 10 (2019) 175-198. doi:10.1146/annurev-chembioeng. URL https://doi.org/10.1146/annurev-chembioeng-

[2] M. Capelot, M. M. Unterlass, F. Tournilhac, L. Leibler, Catalytic control of the vitrimer glass transition, ACS Macro Lett. 1 (7) (2012) 789-792. doi:10.1021/mz300239f. 
[3] P. Zhang, G. Li, Advances in healing-on-demand polymers and polymer composites, Prog. Polym. Sci. 57 (2016) 32-63.

[4] W. Denissen, J. M. Winne, F. E. Du Prez, Vitrimers: permanent organic networks with glass-like fluidity, Chem. sci. 7 (1) (2016) 30-38.

[5] L. Li, X. Chen, K. Jin, J. M. Torkelson, Vitrimers Designed Both to Strongly Suppress Creep and to Recover Original Cross-Link Density after Reprocessing: Quantitative Theory and Experiments, Macromolecules 51 (15) (2018) 5537-5546. doi:10.1021/acs.macromol.8b00922.

[6] C. Yuan, M. Z. Rong, M. Q. Zhang, Z. P. Zhang, Y. C. Yuan, Self-healing of polymers via synchronous covalent bond fission/radical recombination, Chem. Mater. 23 (22) (2011) 5076-5081. doi:10.1021/cm202635w

[7] D. Montarnal, M. Capelot, F. Tournilhac, L. Leibler, Silica-like malleable materials from permanent organic networks, Sci. 334 (6058) (2011) 965968.

[8] S. Billiet, K. De Bruycker, F. Driessen, H. Goossens, V. Van Speybroeck, J. M. Winne, F. E. Du Prez, Triazolinediones enable ultrafast and reversible click chemistry for the design of dynamic polymer systems, Nat. chem. 6 (9) (2014) 815.

[9] W. Denissen, G. Rivero, R. Nicolaÿ, L. Leibler, J. M. Winne, F. E. Du Prez, Vinylogous urethane vitrimers, Adv. Funct. Mater. 25 (16) (2015) 24512457.

[10] R. L. Snyder, C. A. Lidston, G. X. De Hoe, M. J. Parvulescu, M. A. Hillmyer, G. W. Coates, Mechanically robust and reprocessable imine exchange networks from modular polyester pre-polymers, Polym. Chem.

[11] W. Denissen, G. Rivero, R. Nicolaÿ, L. Leibler, J. M. Winne, F. E. Du Prez, Vinylogous urethane vitrimers, Adv. Funct. Mater. 25 (16) (2015) 24512457.

[12] W. Miao, W. Zou, Y. Luo, N. Zheng, Q. Zhao, T. Xie, Chemistry for covalent adaptable polymer networks structural tuning of polycaprolactone based thermadapt shape memory polymer, Polym. Chem. 11 (2020) 13691374 .

[13] X. Chen, M. A. Dam, K. Ono, A. Mal, H. Shen, S. R. Nutt, K. Sheran, F. Wudl, A thermally re-mendable cross-linked polymeric material, Sci. 295 (5560) (2002) 1698-1702.

[14] P. Taynton, H. Ni, C. Zhu, K. Yu, S. Loob, Y. Jin, H. J. Qi, W. Zhang, Repairable woven carbon fiber composites with full recyclability enabled by malleable polyimine networks, Adv. Mater. 28 (15) (2016) 2904-2909. doi:10.1002/adma.201505245. 
[15] Y. Yang, Z. Pei, X. Zhang, L. Tao, Y. Wei, Y. Ji, Carbon nanotube-vitrimer composite for facile and efficient photo-welding of epoxy, Chem. Sci. 5 (9) (2014) 3486-3492.

[16] C. Li, A. Strachan, Molecular dynamics predictions of thermal and mechanical properties of thermoset polymer epon862/detda Polymer 52 (13) (2011) 2920 - 2928. doi:https://doi.org/10.1016/j.polymer. 2011.04 .041

URL http://www.sciencedirect.com/science/article/pii/ S0032386111003338

[17] T. Okabe, Y. Oya, K. Tanabe, G. Kikugawa, K. Yoshioka, Molecular dynamics simulation of crosslinked epoxy resins: Curing and mechanical properties, Eur. Polym. J. 80 (2016) 78 - 88. doi:https://doi.org/10.1016/j.eurpolymj.2016.04.019. URL http://www.sciencedirect.com/science/article/pii/ S0014305716302841

[18] N. Fasanella, V. Sundararaghavan, Atomistic modeling of thermomechanical properties of swnt/epoxy nanocomposites, Model. Simul. Mater. Sci. Eng. 23 (6) (2015) 065003. doi:http://dx.doi.org/10.1088/ 0965-0393/23/6/065003.

URL http://stacks .iop.org/0965-0393/23/i=6/a=065003

[19] V. Varshney, S. S. Patnaik, A. K. Roy, B. L. Farmer, Heat transport in epoxy networks: A molecular dynamics study, Polymer 50 (14) (2009) 3378 - 3385. doi:https://doi.org/10.1016/j.polymer.2009.05.027. URL http://www.sciencedirect.com/science/article/pii/ S0032386109004352

[20] A. Kumar, V. Sundararaghavan, A. R. Browning, Study of temperature dependence of thermal conductivity in cross-linked epoxies using molecular dynamics simulations with long range interactions, Model. Simul. Mater. Sci. Eng. 22 (2) (2014) 025013.

URL http://stacks.iop.org/0965-0393/22/i=2/a=025013

[21] A. Bandyopadhyay, P. K. Valavala, T. C. Clancy, K. E. Wise, G. M. Odegard, Molecular modeling of crosslinked epoxy polymers: The effect of crosslink density on thermomechanical properties, Polymer 52 (11) (2011) 2445 - 2452. doi:https://doi.org/10.1016/j.polymer.2011.03.052. URL http://www.sciencedirect.com/science/article/pii/ S0032386111002606

[22] N. Vu-Bac, M. Bessa, T. Rabczuk, W. K. Liu, A multiscale model for the quasi-static thermo-plastic behavior of highly cross-linked glassy polymers, Macromolecules 48 (18) (2015) 6713-6723.

[23] S. Ciarella, F. Sciortino, W. G. Ellenbroek, Dynamics of vitrimers: Defects as a highway to stress relaxation, Physical review letters 121 (5) (2018) 058003 . 
[24] B. Oyarzún, B. M. Mognetti, Efficient sampling of reversible cross-linking polymers: Self-assembly of single-chain polymeric nanoparticles, Journal of Chemical Physics 148 (11). arXiv:1803.00126, doi:10.1063/1.5020158.

[25] J.-B. Wu, S.-J. Li, H. Liu, H.-J. Qian, Z.-Y. Lu, Dynamics and reaction kinetics of coarse-grained bulk vitrimers: a molecular dynamics study, Physical Chemistry Chemical Physics 21 (24) (2019) 13258-13267.

[26] F. Smallenburg, L. Leibler, F. Sciortino, Patchy particle model for vitrimers, Physical Review Letters 111 (18). doi:10.1103/PhysRevLett. 111.188002 .

[27] F. Sciortino, Three-body potential for simulating bond swaps in molecular dynamics, European Physical Journal E 40 (1). doi:10.1140/epje/ i2017-11496-5.

[28] H. Yang, K. Yu, X. Mu, Y. Wei, Y. Guo, H. J. Qi, Molecular dynamics studying on welding behavior in thermosetting polymers due to bond exchange reactions, RSC Advances 6 (27) (2016) 22476-22487. doi: 10.1039/c5ra26128g

[29] Y. Sun, H. Yang, W. Xia, Y. Guo, Molecular dynamics simulations of surface welding in crosslinked networks with thermally reversible linkages, Applied Surface Science 527 (2020) 146947. doi:10.1016/j.apsusc. 2020. 146947.

[30] H. Yang, K. Yu, X. Mu, X. Shi, Y. Wei, Y. Guo, H. J. Qi, A molecular dynamics study of bond exchange reactions in covalent adaptable networks, Soft Matter 11 (31) (2015) 6305-6317.

[31] J. R. Gissinger, B. D. Jensen, K. E. Wise, Modeling chemical reactions in classical molecular dynamics simulations, Polymer 128 (2017) 211-217.

[32] S. Plimpton, Fast parallel algorithms for short-range molecular dynamics, Tech. rep., Sandia National Labs., Albuquerque, NM (United States) (1993).

[33] A. Rekondo, R. Martin, A. R. de Luzuriaga, G. Cabañero, H. J. Grande, I. Odriozola, Catalyst-free room-temperature self-healing elastomers based on aromatic disulfide metathesis, Mater. Horiz. 1 (2) (2014) 237-240.

[34] A. R. de Luzuriaga, J. M. Matxain, F. Ruipérez, R. Martin, J. M. Asua, G. Cabañero, I. Odriozola, Transient mechanochromism in epoxy vitrimer composites containing aromatic disulfide crosslinks, J. Mater. Chem. C 4 (26) (2016) 6220-6223.

[35] A. R. de Luzuriaga, R. Martin, N. Markaide, A. Rekondo, G. Cabañero, J. Rodríguez, I. Odriozola, Epoxy resin with exchangeable disulfide crosslinks to obtain reprocessable, repairable and recyclable fiber-reinforced thermoset composites, Mater. Horiz. 3 (3) (2016) 241-247. 
[36] V. Sundararaghavan, A. Kumar, Molecular dynamics simulations of compressive yielding in cross-linked epoxies in the context of argon theory, Int.J.Plasticity 47 (2013) 111 - 125. doi:http: //dx.doi.org/10.1016/j.ijplas.2013.01.004.

URL http://www.sciencedirect.com/science/article/pii/ S0749641913000144

[37] P. Dauber-Osguthorpe, V. A. Roberts, D. J. Osguthorpe, J. Wolff, M. Genest, A. T. Hagler, Structure and energetics of ligand binding to proteins: Escherichia coli dihydrofolate reductase-trimethoprim, a drug-receptor system, Proteins: Structure, Function, and Bioinformatics 4 (1) (1988) 31-47.

[38] R. Steudel, Properties of sulfur-sulfur bonds, Angew. Chem.Int.Edit. 14 (10) (1975) 655-664.

[39] N. J. Van Zee, R. Nicolaÿ, Vitrimers: Permanently crosslinked polymers with dynamic network topology, Progress in Polymer Science (2020) 101233.

[40] Y. Zhou, R. Groote, J. G. Goossens, R. P. Sijbesma, J. P. Heuts, Tuning PBT vitrimer properties by controlling the dynamics of the adaptable network, Polymer Chemistry 10 (1) (2019) 136-144. doi:10.1039/ c8py01156g.

[41] K. Yu, A. Xin, Q. Wang, Mechanics of self-healing polymer networks crosslinked by dynamic bonds, Journal of the Mechanics and Physics of Solids 121 (2018) 409-431. doi:10.1016/j.jmps.2018.08.007.

[42] J. Gupta, C. Nunes, S. Jonnalagadda, A molecular dynamics approach for predicting the glass transition temperature and plasticization effect in amorphous pharmaceuticals, Mol. Pharm. 10 (11) (2013) 4136-4145. doi : $10.1021 / \mathrm{mp} 400118 \mathrm{v}$

[43] E. Boulter, M. Cohen, M. Deviney, High-service temperature polyether amide thermoset resins-new materials for electrical insulation and high strength composites, in: Proceedings: Electrical Insulation Conference and Electrical Manufacturing and Coil Winding Conference, IEEE, 1997, pp. 249-253.

[44] M. Ionita, Multiscale molecular modeling of swcnts/epoxy resin composites mechanical behaviour, Compos. B. Eng. 43 (8) (2012) 3491-3496.

[45] A. Shokuhfar, B. Arab, The effect of cross linking density on the mechanical properties and structure of the epoxy polymers: molecular dynamics simulation, J. mol. model. 19 (9) (2013) 3719-3731.

[46] C. Knox, J. Andzelm, J. Lenhart, A. Browning, S. Christensen, High strain rate mechanical behavior of epoxy networks from molecular dynamics simulations, in: 27th Army Science Conference Proceedings, 2010. 
[47] J. M. Barton, A. Deazle, I. Hamerton, B. Howlin, J. Jones, The application of molecular simulation to the rational design of new materials: 2. prediction of the physico-mechanical properties of linear epoxy systems, Polymer 38 (17) (1997) 4305-4310.

[48] L. Zhang, N. De Greef, G. Kalinka, B. Van Bilzen, J.-P. Locquet, I. Verpoest, J. W. Seo, Carbon nanotube-grafted carbon fiber polymer composites: damage characterization on the micro-scale, Compos. B. Eng. 126 (2017) 202-210.

[49] A. H. Barber, S. R. Cohen, S. Kenig, H. D. Wagner, Interfacial fracture energy measurements for multi-walled carbon nanotubes pulled from a polymer matrix, Compos. Sci. Technol. 64 (15) (2004) 2283-2289.

[50] T. Tsuda, T. Ogasawara, F. Deng, N. Takeda, Direct measurements of interfacial shear strength of multi-walled carbon nanotube/peek composite using a nano-pullout method, Compos. Sci. Technol. 71 (10) (2011) 12951300. 